\title{
Enhancing Group Social Perceptiveness through a Swarm-based Decision- Making Platform
}

\author{
David A. Askay \\ California Polytechnic State \\ University \\ daskay@ calpoly.edu
}

\author{
Lynn Metcalf \\ California Polytechnic State \\ University \\ lmetcalf@calpoly.edu \\ Gregg Willcox \\ Unanimous AI \\ gregg@unanimous.ai
}

\author{
Louis Rosenberg \\ Unanimous AI \\ louis@unanimous.ai
}

\begin{abstract}
Swarm Intelligence is natural phenomenon that enables social animals to make group decisions in real-time systems. This process has been deeply studied in fish schools, bird flocks, and bee swarms, where collective intelligence has been observed to emerge. The present paper describes swarm.ai-a collaborative technology that enables swarms of humans to collectively converge upon a decision as a real-time system. Then we present the results of a study investigating if groups working as "human swarms" can amplify their social perceptiveness, a key predictor of collective intelligence. Results showed that groups reduced their social perceptiveness errors by more than half when operating as a swarm. A statistical analysis revealed with $99.9 \%$ confidence that groups working as swarms had significantly higher social perceptiveness than either individuals working alone or through plurality vote.
\end{abstract}

\section{Introduction}

Organizations need to make good decisions [1], and the fast pace of business often requires that they be made quickly and accurately. Yet, this is increasingly challenging in a distributed and datasaturated workplace [2, 3]. In response, many organizations have turned to digital technologies like wikis, business intelligence systems, and crowdsourcing platforms to improve decision-making $[4,5]$. Crowds, in particular, have received much attention for offering insights and making decisions [4, $6,7]$. Indeed, research demonstrates that technologies enabling crowds of individuals to independently provide decisions are able to escape dysfunctional social influences [6]. Yet, crowds also have limitations as they remain susceptible to social influence, which can lead to information cascades that can bias crowdgenerated decisions [8].

While these technologies can provide valuable insights, real-time data, and forecasts, it is often humans working in groups that use this knowledge to make a decision. Indeed, groups are often defined as information processing entities that make decisions [9]. How well groups process information directly links to their performance [10], yet groups often underperform in this regard. To make good decisions, groups members must overcome a host of cognitive biases [11], combat social influence [12], spend time integrating knowledge, and sometimes prevail over oppositional organizational structures [3, 13]. Even achieving that, groups may still not reach consensus or fail to perform adequately.

Consequently, scholarship has long examined the nature of group performance and decision making [11]. Recently, the relationship between collective intelligence and group performance [14] has uncovered promising insights. Between $30-40 \%$ of group performance on a wide range of tasks, from decision-making to mathematical reasoning, can be predicted by a group's collective intelligence [15]. Counterintuitively, the average IQ of group members is only moderately predictive of a group's collective intelligence. Rather, it is the group's average social perceptiveness that is the strongest known predictor of a team's collective intelligence and performance [15]. Groups high in social perceptiveness tend to have higher collective intelligence and are better able to collaborate and coordinate effectively.

As the existence of the collective intelligence factor has been well established in the literature, attention has turned to developing technological tools to facilitate, enhance, and measure the collective 
intelligence of groups [e.g., 4, 14, 16]. Given the important role of social perceptiveness in predicting collective intelligence, scholars have called for research into how social perceptiveness can be altered or enhanced [17].

This study answers this call by using a novel multiagent platform called swarm.ai. This platform is modeled after the swarm intelligence present in natural systems like schools of fish and flocks of birds [e.g., 18, 19]. Swarm.ai enables humans to collaborative in real-time to converge upon a collective decision. We administer a social perception test to 61 groups using both plurality vote and the swarm.ai platform. The results suggest that swarms of humans working together exhibit greater levels of social perceptiveness than either individuals operating alone or group plurality vote. This provides evidence for human swarms to amplify the collective intelligence available to a group during decision making.

\section{Collective Intelligence}

Collective intelligence is studied in various contexts, ranging from colonies of ants [18], to crowds of humans [6], to collections of AI agents [20]. Recent research on the collective intelligence of groups tested 200 teams on a range of different tasks and found that a single, dominate factor explained a large proportion of variance in group scores on a variety of tasks [15]. Moreover, this factor was able to predict performance of more complex tasks in the future. This factor is called collective intelligence and encompasses a group's "capacity to perform across a wide range of tasks". The collective intelligence of groups is conceived as an emergent property resulting from the interaction of bottom-up and top-down group processes [21].

Bottom-up processes are composed of the aggregation of individual group-member attributes that facilitate collaboration. Early research on collective intelligence was premised on the notion that it was a function of the intelligence of individual group members (i.e., IQ). Yet, scholars found only moderate correlations between intelligence of individual members and the collective intelligence of the group [22]. Subsequent research has identified several compositional features of groups that enable collective intelligence. Groups with higher proportion of women, higher average social perceptiveness, and moderate amounts of cognitive diversity have been found to correlate with collective intelligence [15, 16, 23].

The group's average social perceptiveness (sometimes called social sensitivity or social intelligence) is the best known predictor of collective intelligence [17]. It is measured through the Reading the Mind in the Eyes (RME) test [24], which involves viewing photos of people's eyes and identifying which emotions they are expressing. Social perceptiveness is a subset of emotional intelligence skills that pertains to how well one can accurately represent and process information about the mental states of others. Individuals high on this trait can perceive and respond to subtle nonverbal emotional and interpersonal cues, which facilitates interaction and collaboration. This trait predicts $30-40 \%$ of group performance on tasks, even in online environments where there exist limited nonverbal cues and groups only communicate via text [16].

Top-down processes, such as group norms and structures, also enable the emerge of collective intelligence through facilitating effective group interactions. However, research in this area is limited [14]. Lab studies show that collective intelligence is significantly predicted by the total amount of communication in a group and equal distribution of communication among members [16]. However, these findings were not replicated in a field study using gaming teams [25]. Interestingly, collective intelligence even predicts performance in the absence of group communication. A study using a minimumeffort tacit coordination game explored individual decision-making, in which the team gains or loses money as a result of the decisions made by its members [23]. Notably, these decisions were made simultaneously and without communication with each other. Yet, teams with a higher collective intelligence performed better on the task.

Finally, collective intelligence emerges from the interaction of these bottom-up and top-down processes as a part of a system. As Schut [26] explains, collective intelligence emerges in systems when agents can (i) adapt in response to changes via feedback in an uncertain environment, (ii) self-organize autonomously based on local interactions, and (iii) exhibits emergent behavior in which macro-level outcomes are formed through interactions at the micro-level. In other words, collective intelligence is the emergent property of a complex adaptive system (i.e., group) in which agents (i.e. group members) are self-organized through local interactions (i.e., bottomup and top-down processes) and can adapt to changes in the environment. This conceptualization inspires scholars to consider how sociotechnical systems can be designed to enhance groups, such as designing digital environments that structure group interactions or amplifying social cues to enhance emotional intelligence [17].

In summary, groups are central to organizational decision-making, yet are subject to cognitive and 
social influence biases that degrades the quality of decisions. Groups with high collective intelligence are better able to overcome these barriers and perform better at a wide range of tasks, such as decision making. A group's social perceptiveness - the ability to read and respond to subtle nonverbal cues - is the strongest known predictor of collective intelligence. With this in mind, scholars have called for research into how social perceptiveness can be enhanced and how sociotechnical systems might be designed to amplify these traits [17].

This review motivates a study of the potential of groups collaborating as a swarm to amplify the social perceptiveness of the group. Just as collective intelligence emerges from a complex adaptive system, swarm.ai is a platform that enables humans to operate as a real-time system to engage in real-time decision making. This enables groups to engage in human swarming.

\section{Human Swarming}

Studies of collective intelligence technologies predominately focus on crowdsourcing platforms, where crowds of humans contribute a decision (e.g., vote, star-rating, etc.). The platform aggregates and displays the contributions, usually as an average of responses or as the most popular response through a voting paradigm. However, neither of these options are able to capture the real-time dynamics of swarming intelligence observed in nature. Rather, crowd-based platforms generally enable a series of decisions to be made by participants. As these decisions are made serially, earlier decisions by some can influence future decisions of others through social influence and information cascades [8].

By contrast, animals like schools of fish, flocks of birds, and swarms of bees have been shown to deliberate as closed-loop systems that make decisions in parallel with each other - they respond and adapt to subtle feedback cues from other members in real-time to converge upon a decision. For example, schooling fish detect vibrations in the water around them while swarming bees generate and perceive complex body vibrations to deliberate together in systems.

Humans, however, have not evolved with the natural ability to deliberate in real-time, closed-loop swarms. However, this is addressed through the design of swarm.ai, a software platform that enables distributed human groups to connect for the purpose of answering questions, making predictions, and reaching decisions by working together as closed-loop swarms.
As shown in Figure 1, the swarm.ai platform used in this study enables groups of participants to answer questions by collectively moving a graphical puck to select from among a set of alternatives. Each participant provides their individual input by manipulating a small graphical magnet with a mouse, touchpad, or touchscreen. By adjusting the position and orientation of their magnet with respect to the moving puck, participants express their individual intent on the system. The input from each user is not a discrete vote, but a stream of vectors that varies freely over time. Moreover, participants can vary both the direction of their intent and the magnitude of their intent by adjusting the distance between their magnets and the puck.

Because all members of the group can adjust their intent continuously in real-time, the swarm explores the decision-space as a complex adaptive system. Because the graphical puck is in continuous motion, users must continually move their magnets to express their intent. This is significant, for it requires all participants, regardless of group size or composition, to be engaged continuously throughout the deliberation process, evaluating and re-evaluating their intent in real-time. This enables a dynamic negotiation among all members, empowering the group to collectively consider the options and converge on the most agreeable solution.

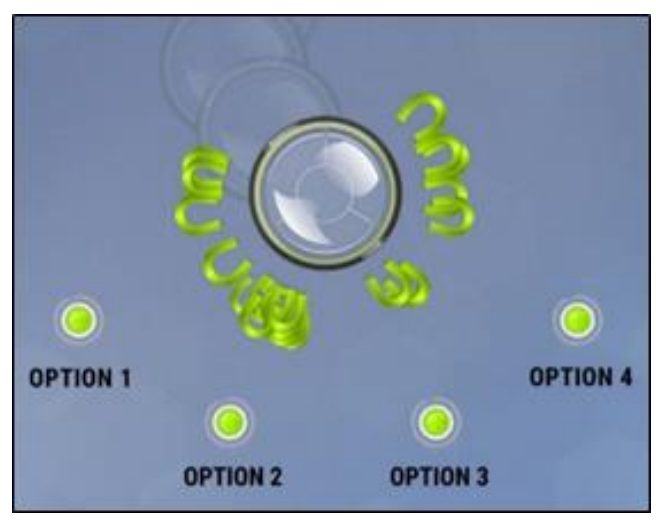

\section{Figure 1. A human swarm choosing between options in real-time}

Thus, like bees vibrating their bodies to express sentiment in a biological swarm, the participants in an artificial swarm must continuously update and express their changing preferences during the decision process or lose their influence over the collective outcome. This is generally referred to as a "leaky integrator" structure and common to swarm-based systems [27]. In addition, algorithms monitor the behaviors of swarm members in real-time, inferring their relative 
conviction based upon their actions and interactions over time. This reveals a range of behavioral characteristics within the swarm population and weights their contributions accordingly, from entrenched participants to flexible participants to fickle participants. Already, human swarms using this platform have significantly increased the predictive accuracy of groups across a variety of tasks, from betting on sporting events to forecasting financial markets [28-32]. Successful swarms have included as low as three to over 40 participants.

There are at least three reasons why swarm.ai is useful in this study. First, collective intelligence emerges from the interactions of a complex adaptive system, which is the kind of system that swarm.ai enables. At the macro-level, as it permits participants to function as a real-time system that responds to changes in the environment (i.e., as the puck moves towards a decision). At the micro-level, it permits individuals to simultaneously and equally provide intent through the direction and magnitude of the graphical magnet and movement of the puck.

Second, swarm.ai can provide a more accurate measure of group-level constructs like group social perceptiveness. A common limitation of group-level research is the reliance of operationalizing a grouplevel construct by aggregating data collected at the individual-level [33]. This is problematic because measurement resides at the individual-level and is therefore unable to capture the underlying group processes of interest [34]. The design of swarm.ai overcomes this limitation because it permits group members to collectively converge upon a single response as a holistic system. The response in inclusive of the group processes present in the team. At the same time, the design of swarm.ai permits a compelling balance between visibility and anonymity that can minimize social influence biases: group members can communicate their opinion visually by moving the puck, yet their identities remain anonymous.

Finally, swarm.ai leverages the sensitivity that humans have to subtle social cues [35]. The movement of the graphical puck amplifies the subtle cues that communicate the intent of the group. This becomes important when recognizing that not all relevant knowledge is explicit. Humans possess tacit knowledge, insights, experiences, and feelings that can be challenging to verbalize with others [36]. Through moving the puck, group members can act upon their tacit knowledge in forming the collective decision.

In sum, swarm.ai is a platform designed to enable humans to engage in the kind of collective intelligence observed in natural swarms. In doing so, swarm.ai offers a novel approach to amplifying and measuring social perceptiveness. To investigate these assertions, we propose the following research question for this study:

RQ1: To what extent does collaborating as a human swarm amplify the social perceptiveness of a group?

\section{Methods}

To assess the ability of human swarms to amplify the social perceptiveness of groups, a large-scale study was conducted across a set of 61 teams, each composed of 3 to 6 members, which were already engaged in a long-term team project together. In total, 302 human subjects participated in this study. All were college students in business, communication studies, and engineering courses, for which the team project was a significant component.

The widely employed "Reading the Mind in the Eyes" (RME) [24] test was used to measure the social perceptiveness of these 61 teams. The test includes 36 questions, each of which provides a facial image restricted to a narrow region around the eyes along with a set of four options that describe the emotion expressed by the person in the image. Participants are asked to identify the emotional state of other people based only on their eyes. An example question from a standard RME test is shown below in Figure 2. As shown, four options are provided, only one of which accurately represents the emotion of the depicted individual.

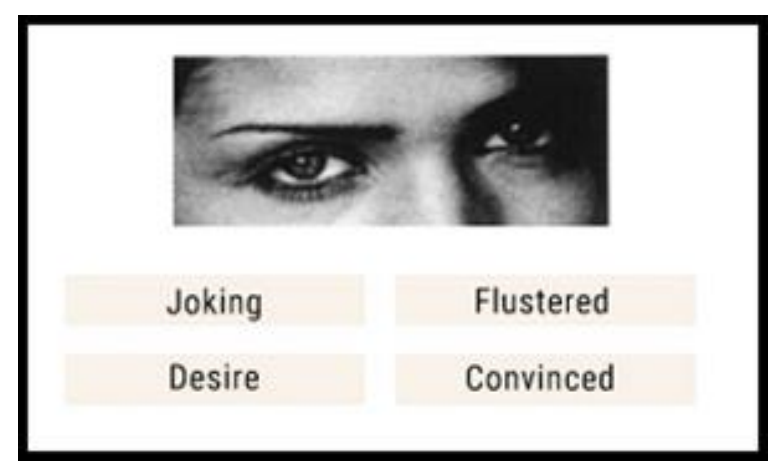

Figure 2. Sample question from RME test

Prior studies have shown that the RME test is a reliable measure of social perceptiveness, with strong internal consistency and test-retest stability [37]. Social intelligence is often described as a person's ability to perceive, interpret, and respond to the intentions, dispositions, and behaviors of others $[38,39]$. These skills are extremely important for effective decision making, especially by problem- 
solving teams, as understanding and/or empathizing with the needs, goals, intentions, and beliefs of others is a fundamental skill required of many critical decisions made by organizations of all sizes [40].

To test whether real-time swarming enabled working groups to amplify their effective social perceptiveness, a two-stage study was employed. First, each of the 302 study participants were administered the RME assessment individually through an online survey. To limit bias and knowledge of correct answers, individual scores were not shared, and discussion of the assessment was discouraged.

In the second stage, each of the 61 teams were administered the RME test through the swarm.ai platform such that the group was tasked with answering each question as a real-time swarm. Team members were discouraged from communicating with each other during the assessment, instead relying only on the closed-loop interaction afforded by the platform (i.e., via pulling the puck). Each team had 60 -seconds to collaboratively coverage upon an answer. Figure 3 below is a snapshot of a participant's screen during a response, which represents the pull of each teammate through a magnet. It should be noted that to discourage conforming to the movement and concentration of magnets rather than the puck, participants did not see the magnets of other participants during the actual swarming session. As the puck moves more slowly and subtly than magnets, this permits time for individuals to consider their position in relation to the overall position of the swarm [17]. Informal discussions with the participants indicated that they enjoyed and were engaged in the experience.

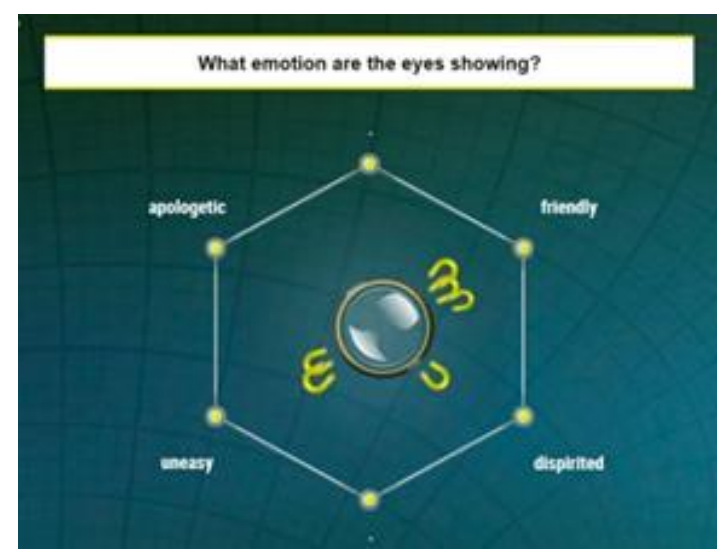

Figure 3. Swarming group responding to
RME question

In sum, the social perceptiveness of groups was measured twice: first through aggregating individual responses for each team and the second through the collective decision of group using the swarm.ai platform.

\section{Data and Analysis}

As noted previously, the RME was administered to 302 individuals across 61 teams, which produced three unique datasets. We received fully completed individual assessments from 266 participants (88\% response rate), totaling over 9,000 item responses. These responses were used to calculate individual $R M E$ scores for each participant. Second, these same responses were aggregated by team to generate a plurality RME score, which was calculated by plurality vote (the most popular answer within a group) for each of the 61 teams. The plurality vote approximates an aggregation based on the voting paradigm embodied in platforms drawing upon the 'wisdom of the crowd.' For questions where the vote was split evenly across multiple answers, a "deadlock" was determined and classified as an incorrect response. This provided a dataset of over 2,500 plurality vote responses to RME assessment questions. Finally, a swarm RME score for each group was calculated from the responses collected through the swarm.ai platform. For questions where the swarm could not converge upon an answer within the 60 second time limit, a "deadlock" was determined and classified as an incorrect response.

During the analysis of the data, it was discovered that responses for question 22 of the swarm RME test were improperly labeled. As a result, all responses for this question were invalid, thus we dropped question 22 from both the individual responses and swarm responses, meaning the maximum value on the RME shifted from 36 to 35 .

\section{Results}

Mean scores and error rates for RME were calculated for the individual, plurality, and swarm generated scores. As shown in Table 1, the average individual RME score was 23.96, which corresponds to an error rate of $31.5 \%$. The average of each team's plurality $R M E$ score was 25.92 , which corresponds to an average error rate of $25.9 \%$. When enabling the teams to work together as a swarm, the average RME score increased to 29.65, which corresponds to an average error rate of $15.3 \%$. In other words, by working together as a swarm, the 61 groups, on average, reduced their error rates by more than half. This supports the notion that working as a swarm can increase the social perceptiveness, and hence the collective intelligence, of groups. 
Table 1. Decision method error rate and confidence interval

\begin{tabular}{|l|l|l|l|l|}
\hline $\begin{array}{l}\text { Testing Method } \\
\text { (Deadlocks as Errors) }\end{array}$ & $\begin{array}{l}\text { Mean \# } \\
\text { Correct }\end{array}$ & $\begin{array}{l}\text { Error } \\
\text { Rate }\end{array}$ & $\begin{array}{l}95 \% \\
\text { Confidence } \\
\text { Interval }\end{array}$ & $\begin{array}{l}95 \% \\
\text { Difference to } \\
\text { Swarm Cl }\end{array}$ \\
\hline Individual Average & 23.96 & $31.54 \%$ & {$[29.9 \%, 33.2 \%]$} & {$[14.0 \%, 18.6 \%]$} \\
\hline Plurality Average & 28.92 & $25.94 \%$ & {$[22.7 \%, 29.2 \%]$} & {$[7.11 \%, 14.4 \%]$} \\
\hline Swarm & 29.65 & $15.29 \%$ & {$[13.1 \%, 17.6 \%]$} & $\mathrm{N} / \mathrm{A}$ \\
\hline
\end{tabular}

Next, the statistical significance of three RME assessment methods were calculated using a 10,000trial bootstrap analysis [41] of the error rate for each method. The $95 \%$ confidence intervals and $p$-values were calculated for the difference between individual RME scores, plurality RME scores, and swarm RME scores. The results show that the swarm significantly outperforms both individual $\left(\mu_{\text {differcence }}=16.3 \%\right.$ error, $p<$ $0.001)$ and plurality scores $\left(\mu_{\text {difference }}=10.7 \%\right.$ error, $\mathrm{p}<$ 0.001 ). The bootstrapped error comparison is shown in Figure 4.

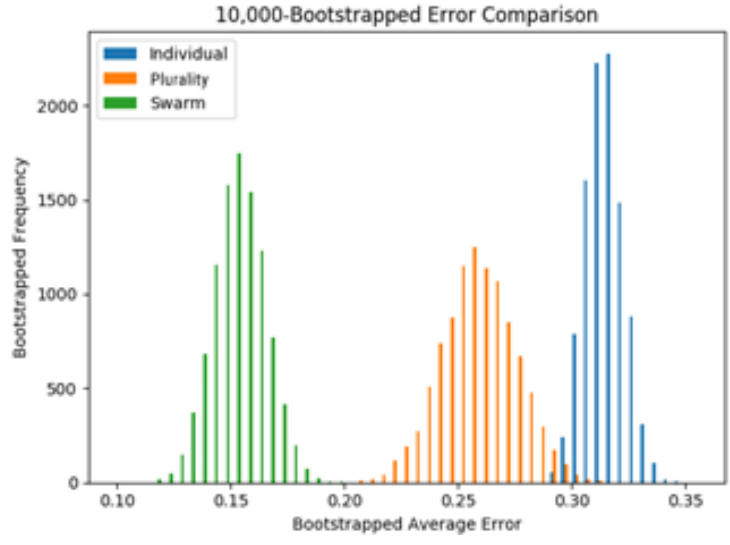

Figure 4. Bootstrapped average error rate

With respect to deadlocks, a comparison was made between the rate of deadlocks determined by plurality vote as compared to the rate of deadlocks reached by swarms. Across the 61 teams, plurality voting resulted in deadlocks in $12 \%$ of questions. Across those same groups, when working together as swarms, the rate of deadlocks dropped substantially to $0.6 \%$ of questions. This is a significant improvement, reducing the need for further steps to resolve undecided groups.

In addition, an analysis was performed that assumed that deadlocked votes were resolved by giving partial credit for tied answers that included a correct response: one-half credit for a two-way tie, one-third credit for a three-way tie, etc. To balance this, deadlocked swarms were given the chance to resolve immediately following a deadlock in another 60 -second swarm, with the answer chosen in this second round selected as the final answer. There were no swarms that deadlocked twice in a row.

As shown in the Table 2 below, when deadlocks were resolved using partial credit, plurality vote had an average RME score of 28.23, or an error rate of $19.3 \%$. When enabling the swarms to work together as real-time systems and resolve their deadlocks in a follow-up swarm, the swarm RME score increased to 29.64 , or an error rate of $15.3 \%$. In other words, even when giving partial credit for deadlocks in group responses determined by plurality vote, the swarm outperformed.

Table 2. Decision method error rate and confidence interval, with deadlocks resolved

\begin{tabular}{|l|l|l|l|l|}
\hline $\begin{array}{l}\text { Testing Method } \\
\text { (Deadlocks } \\
\text { Resolved) }\end{array}$ & $\begin{array}{l}\text { Mean \# } \\
\text { Correct }\end{array}$ & $\begin{array}{l}\text { Error } \\
\text { Rate }\end{array}$ & $\begin{array}{l}\mathbf{9 5 \%} \\
\text { Confidence } \\
\text { Interval }\end{array}$ & $\begin{array}{l}\text { 95\% Difference } \\
\text { to Swarm Cl }\end{array}$ \\
\hline Individual Average & 23.96 & $31.54 \%$ & {$[29.9 \%, 33.2 \%]$} & {$[14.0 \%, 18.6 \%]$} \\
\hline Plurality Average & 28.23 & $19.33 \%$ & {$[17.0 \%, 21.6 \%]$} & {$[1.41 \%, 7.12 \%]$} \\
\hline Swarm & 29.64 & $15.29 \%$ & {$[12.9 \%, 17.5 \%]$} & N/A \\
\hline
\end{tabular}

To assess statistical significance, a bootstrap analysis of the error rate for each method was again performed across 10,000 trials. We find that the swarm outperforms both the plurality vote $\left(\mu_{\text {difference }}=4.0 \%\right.$ error, $p<.002)$ and individuals $\left(\mu_{\text {diffirenece }}=16.3 \%\right.$ error, $\mathrm{p}<.001)$. The bootstrapping of the error rate confidence intervals is shown in Figure 5.

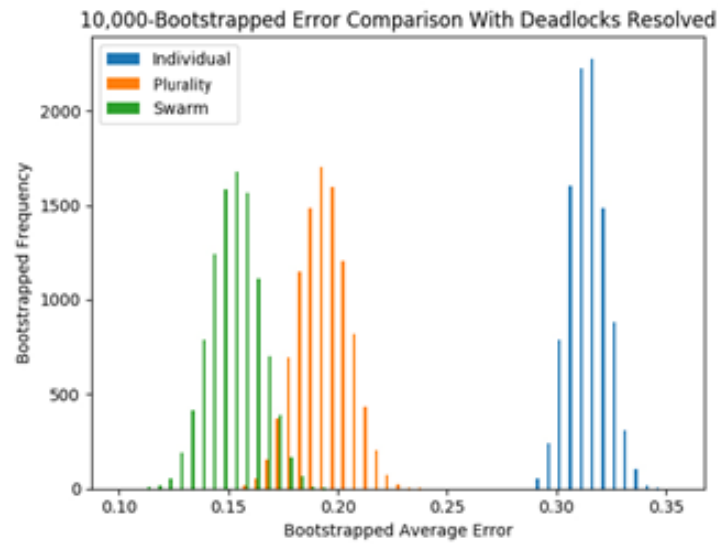

Figure 5. Bootstrapped average error rate

In addition to comparing to the average individual, the swarm can be compared to the full population. On average, swarms are in the $93^{\text {rd }}$ percentile of individuals, indicating that an average 
swarm scores better than $93 \%$ of individuals taking the test alone. The histogram of user performance and average swarm performance is shown in Figure 6.

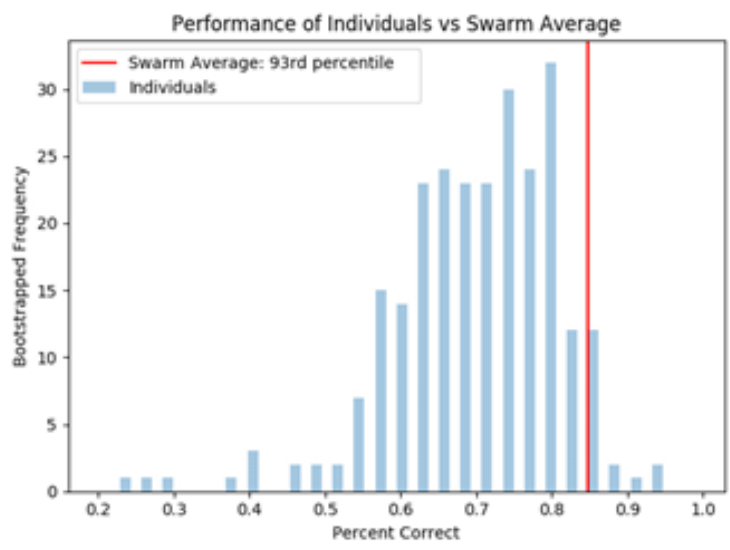

Figure 6. Bootstrapped average error rate

\section{Conclusions}

The purpose of this study was to answer to the call for how digital technologies might be used to amplify the collective intelligence of groups [17]. We addressed this by measuring the group social perceptiveness through plurality vote and human swarming. The results show that social perceptiveness was significantly higher when measured as a swarm than when measured by either individuals or plurality vote, with the swarm score scoring in the 93rd percentile of individuals. Taken together, this indicates human swarms can, in some cases, exhibit greater collective intelligence than a group. Consequently, human swarms offer a compelling and novel way in which organizations might enhance the quality and speed of decision-making. These findings have important implications for theory and practice.

First, the results show that humans swarms collaborating as a real-time system exhibit higher social perceptiveness than when in groups. As social perceptiveness is the greatest known predictor of collective intelligence, this suggests that swarms can perform better on a wide range of tasks and decisions. This interpretation is supported by other successful applications of human swarms to make surprisingly accurate decisions, from predicting sporting event outcomes to forecasting financial markets [28-32]. Future research can examine further the kinds of decisions and tasks that are best suited for human swarming. For example, while crowd-based platforms often excel in divergent thinking (e.g., the number and diversity of ideas generated), swarming may be more appropriate for convergent thinking (e.g., narrowing down and selecting a single idea). Indeed, this provides direction for how human swarms might complement crowd-based decision platforms.

An alternate interpretation of the results is that group social perceptiveness is more accurately measured by a swarm than through aggregation. This is an equally interesting interpretation with promising implications for the measurement of multilevel constructs such as groups, teams, organizational subunits, and entire organizations [42]. Future research can investigate swarming as a potential for more accurate method of multilevel measurement.

Second, the design of swarm.ai also provides interesting implications. Swarm.ai is a sociotechnical system that amplifies subtle social signals such that the entire group benefits from a greater level of social perceptiveness. This is important as there is limited research on how theory-of-mind abilities like emotional intelligence and social perceptiveness might be trained or enhanced [17]. These findings suggest human swarming might be developed as a tool or intervention for training people to enhance their emotional intelligence.

Thirdly, the findings suggest that a biomimicry approach to designing mass collaborative systems may be fruitful. While swarm intelligence is currently used in areas like social learning [43] and navigation of robots [44], relatively little research has been done to extend this concept further into human decision making. For example, future research may investigate how swarming systems might enable human-AI collaboration [45], whereby human and non-human agents collaborate together to converge upon optional decisions. Additionally, swarming may help to break through functional limits of how large a decisionmaking group can be. Research shows that after about 12 group members, coordination costs prevent groups from functioning effectively [46]. By contrast, there are few, if any, limitations to how many people can participate in a swarm - swarm.ai has enabled over 40 participants to collaborate in real-time. Human swarming systems may help to achieve what Malone calls a 'supermind,' whereby dozens, potentially thousands, of people can simultaneously and successfully collaborate [47]. The design of such collaborative platforms and business intelligence systems might be informed from the swarm intelligence of natural systems.

Fourth, the findings suggest that there might limits surrounding when groups need to share knowledge when making a decision. Indeed, prior studies reveal that high levels of collective intelligence can be found in teams that do not explicitly communicate with each other [23]. Additionally, others found that network 
patterns of interaction among a team, more so than the content of communication, can predict group performance $[35,48]$. This study contributes to this literature by adding that human swarms can be successful in an environment with limited linguistic interaction, instead relying on the movement of a graphical puck to subtly communicate intent. These findings introduce important questions about the nature and needs of decision making and information sharing.

In addition to these implications, it is important to note the limitations of the study. As described earlier, it may be that the increased social perceptiveness is the result of greater accuracy of this construct. Also, each participant viewed the RME questions twice-once during the individual survey and once during the swarm. While 1-3 weeks lapsed between these two assessments and participants did not receive feedback on their responses, it is possible that there might be a recall bias that can explain the increased performance in the swarm.

In conclusion, this study contributes to the research on collective intelligence and group decision making by showing how human swarms can, in some instances, outperform groups on a social perceptiveness task. The results demonstrate how humans collaborating as a real-time system can amplify the collective intelligence available to group and suggests that swarms will perform better on a variety of tasks. Future research investigating the role of human swarms in the future of work are needed.

\section{References}

[1] R. Sharma, and S. Mithas, "Transforming decisionmaking processes: a research agenda for understanding the impact of business analytics on organisations," European Journal of Information Systems, vol. 23, no. 4, 2014, pp. 433-441.

[2] The Workplace Evolution, Harvard Business Review Analytic Services, 2018.

[3] How Collaboration Wins: Leadership, Benefits, and Best Practices, Harvard Business Review Analytic Services, 2017.

[4] E. Bonabeau, "Decisions 2.0: The power of collective intelligence," MIT Sloan management review, vol. 50, no. 2, 2009, pp. 45-52.

[5] V. Trieu, "Getting value from Business Intelligence systems: A review and research agenda," Decision Support Systems, vol. 93, 2017, pp. 111-124.
[6] J. Surowiecki, The wisdom of crowds, Anchor, 2005.

[7] T. W. Malone, R. Laubacher, and C. Dellarocas, "The collective intelligence genome," MIT Sloan Management Review, vol. 51, no. 3, 2010, pp. 21-31.

[8] L. Muchnik, S. Aral, and S. J. Taylor, "Social influence bias: A randomized experiment," Science, vol. 341, no. 6146, 2013, pp. 647-651.

[9] V. B. Hinsz, R. S. Tindale, and D. A. Vollrath, "The emerging conceptualization of groups as information processors," Psychological bulletin, vol. 121, no. 1, 1997, pp. 43-64.

[10] J. R. Mesmer-Magnus, and L. A. DeChurch, "Information sharing and team performance: A metaanalysis," Journal of Applied Psychology, vol. 94, no. 2, 2009, pp. 535-546.

[11] N. L. Kerr, and R. S. Tindale, "Group performance and decision making," Annual Review of Psychology, vol. 55, 2004, pp. 623-655.

[12] J. K. Esser, "Alive and well after 25 years: A review of groupthink research," Organizational behavior and human decision processes, vol. 73, no. 2-3, 1998, pp. 116-141.

[13] E. J. Russo, and P. J. Schoemaker, Decision traps: Ten barriers to brilliant decision-making and how to overcome them, Doubleday, New York, 1989.

[14] A. W. Woolley, I. Aggarwal, and T. W. Malone, "Collective intelligence and group performance," Current Directions in Psychological Science, vol. 24, no. 6, 2015, pp. 420-424.

[15] A. W. Woolley, C. F. Chabris, A. Pentland, N. Hashmi, and T. W. Malone, "Evidence for a collective intelligence factor in the performance of human groups," Science, vol. 330, no. 6004, 2010, pp. 686688.

[16] D. Engel, A. W. Woolley, L. X. Jing, C. F. Chabris, and T. W. Malone, "Reading the mind in the eyes or reading between the lines? Theory of mind predicts collective intelligence equally well online and face-to-face," PloS one, vol. 9, no. 12, 2014.

[17] A. W. Woolley, I. Aggarwal, and T. W. Malone, "Collective intelligence in teams and organizations," Handbook of collective intelligence, T. W. Malone and 
M. S. Bernstein, eds., pp. 143-168, MIT Press, Cambridge, MA, 2015.

[18] E. Bonabeau, M. Dorigo, and G. Theraulaz, Swarm intelligence: from natural to artificial systems, Oxford University Press, New York, NY, 1999.

[19] S. Garnier, J. Gautrais, and G. Theraulaz, "The biological principles of swarm intelligence," Swarm Intelligence, vol. 1, no. 1, 2007, pp. 3-31.

[20] J. Hernández-Orallo, The measure of all minds: evaluating natural and artificial intelligence, Cambridge University Press, New York, NY, 2016.

[21] J. Salminen, "Collective intelligence in humans: A literature review," in Collective Intelligence 2012, Boston, MA, 2012.

[22] D. J. Devine, and J. L. Philips, "Do smarter teams do better: A meta-analysis of cognitive ability and team performance," Small Group Research, vol. 32, no. 5, 2001, pp. 507-532.

[23] I. Aggarwal, A. W. Woolley, C. F. Chabris, and T. W. Malone, "Cognitive diversity, collective intelligence, and learning in teams," in Proceedings of Collective Intelligence 2015, Santa Clara, CA, 2015.

[24] S. Baron-Cohen, S. Wheelwright, J. Hill, Y. Raste, and I. Plumb, "The "Reading the Mind in the Eyes" Test revised version: a study with normal adults, and adults with Asperger syndrome or highfunctioning autism," The Journal of Child Psychology and Psychiatry and Allied Disciplines, vol. 42, no. 2, 2001, pp. 241-251.

[25] Y. J. Kim, D. Engel, A. W. Woolley, J. Y.-T. Lin, N. McArthur, and T. W. Malone, "What Makes a Strong Team?: Using Collective Intelligence to Predict Team Performance in League of Legends." pp. 2316-2329.

[26] M. C. Schut, "On model design for simulation of collective intelligence," Information Sciences, vol. 180 , no. 1,2010 , pp. 132-155.

[27] H. Jaeger, M. Lukoševičius, D. Popovici, and U. Siewert, "Optimization and applications of echo state networks with leaky-integrator neurons," Neural networks, vol. 20, no. 3, 2007, pp. 335-352.

[28] L. Rosenberg, N. Pescetelli, and G. Willcox, "Artificial Swarm Intelligence amplifies accuracy when predicting financial markets," in Proceedings of the IEEE 8th Annual Ubiquitous Computing, Electronics and Mobile Communication Conference (UEMCON), New York, NY, 2017, pp. 58-62.

[29] L. Rosenberg, and G. Willcox, "Artificial Swarms find Social Optima," in IEEE Conference on Cognitive and Computational Aspects of Situation Management (CogSIMA), Boston, MA, 2018, pp. 174-178.

[30] L. Rosenberg, “Artificial Swarm Intelligence vs human experts," in International Joint Conference on Neural Networks (IJCNN), Vancouver, BC, 2016, pp. 2547-2551.

[31] L. Rosenberg, D. Baltaxe, and N. Pescetelli, "Crowds vs swarms, a comparison of intelligence," in Swarm/Human Blended Intelligence Workshop (SHBI), Cleveland, OH, 2016, pp. 1-4.

[32] L. Rosenberg, "Human Swarms, a real-time method for collective intelligence," in Proceedings of the European Conference on Artificial Life (ECAL), York, UK, 2015, pp. 658-659.

[33] D. M. Rousseau, "Issues of level in organizational research: Multi-level and cross-level perspectives," Research in Organizational Behavior, vol. 7, no. 1, 1985, pp. 1-37.

[34] B. L. Kirkman, P. E. Tesluk, and B. Rosen, "Assessing the incremental validity of team consensus ratings over aggregation of individual-level data in predicting team effectiveness," Personnel Psychology, vol. 54, no. 3, 2001, pp. 645-667.

[35] A. Pentland, "On the collective nature of human intelligence," Adaptive Behavior, vol. 15, no. 2, 2007, pp. 189-198.

[36] I. Nonaka, "A dynamic theory of organizational knowledge creation," Organization Science, vol. 5, no. 1, 1994, pp. 14-37.

[37] M. Vellante, S. Baron-Cohen, M. Melis, M. Marrone, D. R. Petretto, C. Masala, and A. Preti, "The "Reading the Mind in the Eyes" test: systematic review of psychometric properties and a validation study in Italy," Cognitive Neuropsychiatry, vol. 18, no. 4, 2013, pp. 326-354.

[38] S. T. Fiske, and S. E. Taylor, Social cognition: From brains to culture, 3rd ed., SAGE, Los Angeles, CA, 2017. 
[39] Z. Kunda, Social cognition: Making sense of people, MIT Press, Cambridge, MA, 1999.

[40] C. D. Frith, and T. Singer, "The role of social cognition in decision making," Philosophical Transactions of the Royal Society B: Biological Sciences, vol. 363, no. 1511, 2008, pp. 3875-3886.

[41] C. Z. Mooney, and R. D. Duval, Bootstrapping: A nonparametric approach to statistical inference, SAGE, Newbury Park, CA, 1993.

[42] P. D. Bliese, D. Chan, and R. E. Ployhart, "Multilevel methods: Future directions in measurement, longitudinal analyses, and nonnormal outcomes," Organizational Research Methods, vol. 10, no. 4, 2007, pp. 551-563.

[43] R. Cheng, and Y. Jin, "A social learning particle swarm optimization algorithm for scalable optimization," Information Sciences, vol. 291, 2015, pp. $43-60$.

[44] R. Fujiwara, T. Kano, and A. Ishiguro, "Selfswarming robots that exploit hydrodynamical interaction," Advanced Robotics, vol. 28, no. 9, 2014, pp. 639-645.

[45] P. R. Daugherty, and H. J. Wilson, Human+ Machine: Reimagining Work in the Age of AI, Harvard Business Review Press, Boston, MA, 2018.

[46] B. C. Poulton, and M. A. West, "The determinants of effectiveness in primary health care teams," Journal of Interprofessional Care, vol. 13, no. 1, 1999, pp. 718.

[47] T. W. Malone, Superminds: The Surprising Power of People and Computers Thinking Together, Little, Brown and Company, New York, NY, 2018.

[48] A. Pentland, "The new science of building great teams," Harvard Business Review, vol. 90, no. 4, 2012, pp. 60-69. 